\title{
A comparison of machine learning algorithms for forecasting indoor temperature in smart buildings
}

\author{
Sadi Alawadi ${ }^{1}$ - David Mera ${ }^{2}$ - Manuel Fernández-Delgado ${ }^{2}$. \\ Fahed Alkhabbas ${ }^{1} \cdot$ Carl Magnus Olsson $^{1} \cdot$ Paul Davidsson ${ }^{1}$
}

Received: 28 August 2019 / Accepted: 10 January 2020 / Published online: 24 January 2020

(c) The Author(s) 2020

\begin{abstract}
The international community has largely recognized that the Earth's climate is changing. Mitigating its global effects requires international actions. The European Union (EU) is leading several initiatives focused on reducing the problems. Specifically, the Climate Action tries to both decrease EU greenhouse gas emissions and improve energy efficiency by reducing the amount of primary energy consumed, and it has pointed to the development of efficient building energy management systems as key. In traditional buildings, households are responsible for continuously monitoring and controlling the installed Heating, Ventilation, and Air Conditioning (HVAC) system. Unnecessary energy consumption might occur due to, for example, forgetting devices turned on, which overwhelms users due to the need to tune the devices manually. Nowadays, smart buildings are automating this process by automatically tuning HVAC systems according to user preferences in order to improve user satisfaction and optimize energy consumption. Towards achieving this goal, in this paper, we compare 36 Machine Learning algorithms that could be used to forecast indoor temperature in a smart building. More specifically, we run experiments using real data to compare their accuracy in terms of R-coefficient and Root Mean Squared Error and their performance in terms of Friedman rank. The results reveal that the ExtraTrees regressor has obtained the highest average accuracy $(0.97 \%)$ and performance $(0,058 \%)$ over all horizons.
\end{abstract}

Keywords Smart buildings · Time series prediction · Energy efficiency · Machine Learning $\cdot$ Internet of Things

Sadi Alawadi

sadi.alawadi@mau.se

Extended author information available on the last page of the article 


\section{Introduction}

Climate change is one of the bigger challenges we face today, needing immediate and long-term action. In general, climate change affects all regions of the world-posing a threat to global economy, holding negative environmental effects, and bringing worrying health implications. These growing threats require international action to mitigate and minimize their negative effects. Initiatives such as the European Climate Action outline the recorded negative effects of climate change and list urban infrastructure as the key to effectively working towards the goals set forth by the European Union (EU), who is currently responsible for $71 \%$ of global gas emissions and thus has a vital role to play [60]. To achieve this, the European Climate Action initiative aims to both decrease EU greenhouse gas emissions and improve energy efficiency by reducing the amount of primary energy consumed. It also aims to find sustainable solutions from an environmental as well as an economic standpoint.

Within smart buildings, the automation of existing residential as well as commercial buildings (built prior to modern low- or zero-energy buildings) plays a significant role, as such buildings make up the majority of energy consumption. The EU has pointed to the development of efficient building energy management systems as key to achieving the identified objectives due to the fact that buildings account for $40 \%$ of energy consumption and $36 \%$ of total $\mathrm{CO}_{2}$ emissions within the $\mathrm{EU}[3,72]$. The majority of energy in those buildings is consumed by Heating, Ventilation, and Air Conditioning (HVAC) systems, which have strong impact on households comfort as well as on the environment [29].

Increasing affordability as well as rising temperatures have meant that HVAC systems are increasingly being used to improve comfort and thus quality of everyday life. At the same time, such systems can quickly consume a considerable amount of energy. Particularly for systems with limited intelligent behavior, energy efficiency is not emphasized, and simple matters quickly add up to energy waste-such as a household forgetting to turn off an air conditioner before going to work or systems not adapting when the weather changes by, for instance, turning off when not needed.

In traditional (non-smart) buildings, users (residents) are responsible for monitoring and controlling available devices. However, contemporary smart buildings are increasingly equipped with Internet of Things (IoT) devices and objects such as sensors, actuators, connected air conditioners, and heaters. In such buildings, unlike traditional buildings, IoT devices collaborate to automatically adjust temperature and optimize the use of HVAC systems, for instance, by forecasting the indoor temperature and generating plans for tuning HVAC devices to optimize energy consumption.

Previous studies have shown that Machine Learning (ML) algorithms can be exploited to model most of the systems in the smart buildings. In particular, ML can be used to model the current HVAC systems [23] to improve energy efficiency and reduce consumption in such buildings. ML is a sub-field of Artificial Intelligence (AI) that combines a set of mathematical algorithms to give systems the ability to learn automatically and improve the experience without being explicitly programmed [4]. Nowadays, ML is widely used in many fields, including health care, public transportation, and smart cities systems $[2,16,45]$. ML is divided into several categories based on the learning method, such as supervised, semi-supervised, unsupervised, and 
enforced learning. In this paper we will be using supervised learning, which is divided into two main branches; classification [7,28,62] and regression $[1,27]$ depending on the problem that needs to be solved. In our case we will be using regression to forecast the indoor temperature.

In this paper, we describe an experiment that compares 36 offline ML algorithms used for forecasting the indoor temperature for three consecutive hours in a smart building. A real dataset was collected from the CiTIUS research center and the closest weather station sensor measurements that belongs to different winter periods with different weather conditions as reported in Table 1. All algorithms were evaluated based on their accuracy, performance, and robustness to weather changes. The main aim of this study is to find the most suitable ML algorithm in terms of the performance and robustness that can be integrated into building management systems (BMS) to improve building energy efficiency. Specifically to tune HVAC system parameters taking into consideration user comfort levels and reduction of energy consumption. We concluded that increasing the forecasting time does not decrease the accuracy of the best model. Moreover, we found that the difference between the obtained results for three consecutive forecasting hours is insignificant (around 0.01) for both R-coefficient and RMSE; This means that the increase of the horizon does not rapidly affect the accuracy of extraTrees.

The remainder of this paper is organized as follows. In the next Sect. 2, we review existing studies to forecast the building's indoor temperature using different ML algorithms. In Sect. 3, we describe the dataset we used to develop the experiments and explain the ML algorithms used to develop the experiments. Section 4 shows the results and discussion. Finally, Sect. 5 draws the conclusions and outlines of the future work.

\section{Related research}

Previous studies have determined that the HVAC systems have the highest energy demand in a building. Therefore, managing HVAC systems in current buildings should be addressed to improve energy efficiency by improving energy plans. In particular, developing a ML model that considers the surrounding factors is necessary to configure the best HVAC system parameters. Those parameters have a relevant impact on both energy consumption and user comfort [23]. The ML model Artificial Neural Network (ANN) is widely used for indoor temperature forecasting. Nivine et al. [8] proposed a new approach to forecast the indoor temperature up to $4 \mathrm{~h}$ based on ANN by considering the outdoor parameters. Further, Kwok et al. [44] modulated the cooling load in a smart building by incorporating a Neural Network (NN) into an intelligent model that allows forecasting and examining the energy demand of the building as well as determining the critical factors that impact on energy consumption. The study reveals that the building occupancy is a significant factor in forecasting the cooling load of the HVAC system. In [54], the authors studied the impact of both users' activities and their behaviors on potential energy saving in smart buildings. The authors classified the user as the most important factor and divided the user impact on energy demands into three main subsystems: HVAC, light, and plug load systems. 
Moreover, Varick et al. [25] used real-time data to study building occupancy and its influence on energy saving. They proposed an occupancy model that could be successfully integrated into the HVAC system in the building through Markov Chains. The study revealed that this model could annually save $42 \%$ of consumed energy. Zhao [62] argued that external factors also have a significant influence on a building's energy performance through reviewing various energy forecast methods implemented into ML algorithms and studying the engineering and statistical techniques utilized to predict a building's energy consumption [72].

In [48], a new model was developed based on Support Vector Regression (SVR) to predict the hourly cooling load inside office buildings. The model's hyper-parameters were tuned to get the best temperature forecast. The study compared the developed model with the classical multi-layer perceptron neural network (MLP) and showed that the SVR outperforms the MLP in both accuracy and mean squared error (MSE). In addition, Dong [21] examined the feasibility of forecasting building energy consumption by applying SVR for regression and determined the impact of different SVR parameters on the prediction accuracy. The study exposes that SVR obtained the highest accuracy compared with other relevant research approaches using genetic programming and neural networks. Previous studies addressed external weather conditions and their influence on indoor temperature through autoregressive model (ARX) and autoregressive moving average model (ARMAX). The selection of the suitable structure of both models has been determined to obtain the best prediction accuracy. These models can become a flexible controller because of their dynamic structure, which permits to increase the user's comfort level inside the building and to improve the energy efficiency of HVAC systems [58]. The outcomes exhibited that the ARX model achieved the best forecasting accuracy.

Sülo et al. [67] developed a deep learning model to predict the energy consumption value of each building resides in the City University of New York (CUNY) campuses. Each one of those buildings has different energy expenditures. Where, the optimal conditions and forecasting the future energy usage of those buildings have been investigated to determine the loss of energy, using long short-term memory (LSTM) Neural Networks models. The experiments were conducted using time series data that were collected from several campuses of CUNY. Furthermore, Xu et al. [18] used an LSTM deep learning model to forecast the indoor temperature for 5 and $30 \mathrm{~min}$ in advance. The LSTM model was compared to three standard ML models Back Propagation Neural Network (BPNN), Support Vector Machine (SVM) and Decision Tree (DT), which it outperformed. In [42] Jin et al. used deep learning to forecast the optimal indoor temperature with the aim to adjust the air conditioner automatically without any user interference.

Abdullatif et al. [10] proposed a cooling load forecasting model for buildings, utilizing the generalized regression neural network (GRNN) taking into consideration the building orientational characteristics and occupancy in order to optimize the thermal energy storage of the HVAC.

Catalina [14] developed polynomial regression models based on neural networks to predict the monthly heating demand for residential buildings, considering the residential constructional structure. Catalina used 270 different scenarios to validate the developed models to find the best approach. Several other recent investigations 
proposed models using different ML algorithms for forecasting a building's energy consumption $[24,53,61,72]$. In these studies, various external factors were considered, such as building structure, orientation, isolation, and environmental variables. The statistical results showed that these factors have a significant influence on indoor temperature prediction and energy consumption in a building. Kangji et al. [47] developed a GA-ANFIS model to predict the indoor building temperature. This approach obtained the optimal configuration of subtractive clusters, using a genetic algorithm (GA) to optimize the fuzzy if-then rule base. The adaptive network-based fuzzy inference system (ANFIS) adjusted the premise and subsequent parameters to match the training data. The results showed that GA-ANFIS obtained higher performance levels compared to neural networks in terms of prediction accuracy.

Recently, Rodríguez-Mier et al. [59] used FRULER-GFS (fuzzy rule learning through evolution for regression-genetic fuzzy system) to develop a rule-based model for forecasting indoor temperature. The knowledge bases learned by FRULER include Takagi-Sugeno-Kang fuzzy rules that correctly predict the temperature dynamics measured by several different predictors obtained from both inside and outside the building. The experiment results demonstrated that FRULER-GFS had the best accuracy rate compared with ElasticNet and random forest regressors [59].

Further, Doukas et al. [22] developed an integrated decision support system based on rule sets. Their study aimed at improving the energy management system of a building. Their system allowed central control over energy consumption in the building, which made it exceptionally flexible. Furthermore, they created a reliable energy profile using expert knowledge in the system. The HVAC control optimization (On/Off) provided the system with the capability to recognize and discard any wrong decision. The study confirmed that expert experience has a notable impact on improving the building energy management system.

When reviewing previous studies on improving energy efficiency of HVAC systems in smart buildings, none has compared a large set of ML algorithms to predict the indoor temperature of buildings. This study provides a baseline for future studies on forecasting the indoor temperatures in smart buildings using ML algorithms. All models developed have been trained using the same settings for different weather conditions to check the robustness and the performance of these algorithms.

\section{Experiments}

\subsection{Experiments setups}

As part of the European OPERE project [26], which aims at improving the energy management system of the Universidade de Santiago de Compostela (USC), the USC has deployed sensors in 45 university buildings. In this paper, we conducted experiments considering one of those smart buildings, called Centro Singular de Investigación en Tecnoloxías Intelixentes (CiTIUS), using a medium-sized sensor network. The network collects and reports sensor readings as illustrated in Table 1. It produces 667 signals every $10 \mathrm{~s}$. 
Table 1 Pattern features, where (*) represents features from CiTIUS, and (+) symbolizes features from Meteogalicia

\begin{tabular}{|c|c|c|c|}
\hline Features & Abbr. & Type & Description \\
\hline Underfloor Heating Status * & UHS & Binary & $\begin{array}{l}\text { Status of the underfloor heating } \\
\text { system (on/off) in the office }\end{array}$ \\
\hline $\begin{array}{l}\text { Underfloor Heating Temperature } \\
*\end{array}$ & UHT & Continuous & $\begin{array}{l}\text { Temperature of the water linked to } \\
\text { the underfloor heating system }\end{array}$ \\
\hline Air Condition Status * & ACS & Binary & $\begin{array}{l}\text { Status of the air conditioning system } \\
\text { (on/off) in the office }\end{array}$ \\
\hline Air Conditioning Temperature* & $\mathrm{ACT}$ & Continuous & $\begin{array}{l}\text { The desired temperature of the } \\
\text { central air conditioning system. }\end{array}$ \\
\hline Air Conditioning Humidity * & $\mathrm{ACH}$ & Continuous & $\begin{array}{l}\text { The percentage of the humidity } \\
\text { attached to central air conditioning } \\
\text { flow }\end{array}$ \\
\hline Humidity + & OutH & Continuous & $\begin{array}{l}\text { Degree of the outdoor relative } \\
\text { humidity }\end{array}$ \\
\hline Temperature + & OutT & Continuous & Outdoor temperature \\
\hline Solar radiation + & SR & Continuous & Level of solar radiation \\
\hline Indoor temperature * & $\mathrm{T}$ & Continuous & $\begin{array}{l}\text { Indoor temperature in one particular } \\
\text { office }\end{array}$ \\
\hline Previous indoor temperature $*$ & $\mathrm{~T}-1$ & Continuous & $\begin{array}{l}\text { The actual office temperature in a } \\
\text { specific time period }(1,2 \text { and } 3 \mathrm{~h})\end{array}$ \\
\hline
\end{tabular}

The dataset we used to develop the experiments composed both the sensor measurements linked to the CiTIUS HVAC system and weather data collected from the closest Meteogalicia weather station. The CiTIUS building has two functionality modes: winter and summer modes. The dataset patterns were retrieved every 10 min during two different time periods: from October 1, 2015, to March 31, 2016 (26,321 patterns), and from November 1, 2016, to January 31, 2017 (13,083 patterns). Both periods correspond to the HVAC winter working mode, which has the highest energy demand. It must be noted that the second period corresponds to an unusually dry winter season in Galicia. Thus, the weather conditions in both periods are different enough.

Each dataset pattern comprises 10 features, seven of them are provided by the CiTIUS and the rest by Metogalicia weather station. Each variable indicates a measurable phenomenon that can reduce the energy demand for heating and cooling the building; these features are described in Table 1.

\subsection{Machine learning algorithms}

In this paper, we compared 36 batch learning algorithms belonging to 20 different families (as listed in Table 2) [27]. All algorithms were selected based on the recommendation of the study conducted by Sirsat et al. [63]. The main purpose of the experiment was to identify which of those algorithms is the most accurate to forecast the indoor temperature of the studied building. The majority of the algorithms were 
selected from the Classification and Regression Training package ${ }^{1}$ in the statistical computing language $\mathrm{R}^{2}$

The experiments for each algorithm were repeated 10 times using different seeds generated randomly. The data partitions were generated randomly in such a way that $70 \%, 15 \%$, and $15 \%$ of the patterns were used for training, validating, and testing the models, respectively. For each algorithm, the hyper-parameters were tuned using the values reported in Table 3. The selected final values for the hyper-parameter are those that maximize the average performance over the validation sets.

Furthermore, we implemented three more popular methods using other platforms: support vector regression (SVR) using the LibSVM library was implemented in $\mathrm{C}++,{ }^{3}$ and Generalized Regression Neural Network (GRNN) and Extreme Learning Machine (ELM) with Gaussian kernels were both implemented in MATLAB. ${ }^{4}$ Moreover, we trained the regressors by exploiting the values reported in Table 3, and stated in the $\mathrm{R}$ package documentation to tune the algorithm hyper-parameters.

We then evaluated the tested algorithms' performance using Pearson correlation (Rcoefficient) that falls between $(+1,-1)$, shown in Eq. 1, and the Root Mean Squared Error (RMSE), shown in Eq. 2.

$$
\rho(\hat{Y}, Y)=\frac{1}{N-1} \sum_{i=1}^{N}\left(\frac{\overline{\hat{Y}_{i}-\mu_{\hat{Y}}}}{\sigma_{\hat{Y}}}\right)\left(\frac{Y_{i}-\mu_{Y}}{\sigma_{Y}}\right)
$$

where $\mu_{\hat{Y}}$ and $\sigma_{\hat{Y}}$ are the mean and standard deviation of the predicted temperature $\hat{Y}$, while $\mu_{Y}$ and $\sigma_{Y}$ are the mean and the standard deviation of the real temperature $Y$, and $N$ is the number of test patterns.

$$
R M S E=\sqrt{\frac{1}{N} \sum_{i=1}^{N}\left(\hat{Y}_{i}-Y_{i}\right)^{2}}
$$

The final regressor performance matrices were computed in the developed experiments by taking the average of both RMSE and R-coefficient over the 10 repetitions.

\section{Results and discussion}

Satisfying users by achieving and maintaining their comfort levels and optimizing energy consumption inside buildings should be core aspects when realizing smart buildings. This requires developing accurate and reliable HVAC systems that are automatically adaptable to different weather conditions. Towards achieving this goal, we compared $36 \mathrm{ML}$ algorithms, over a real data set, to predict the indoor temperature in the CiTIUS office. The results can be utilized to generate energy plans that tune

\footnotetext{
1 http://topepo.github.io/caret/train-models-by-tag.html.

2 http://r-project.org.

3 https://www.csie.ntu.edu.tw/ cjlin/libsvm.

4 http://mathworks.com.
} 
Table 2 Regressors considered in this work, grouped by families

\begin{tabular}{|c|c|c|}
\hline No. & Family & Regressors \\
\hline 1 & Bayesian models & $\begin{array}{l}\text { Bayesian GLM (Bayesglm) [36] } \\
\text { Bayesian regularized neural network (Brnn) }[30,49]\end{array}$ \\
\hline 2 & Bagging ensembles & $\begin{array}{l}\text { Bagging ensemble of conditional inference } \\
\text { regression trees (Bag) [11] } \\
\text { Bagged multivariate adaptive regression (BagEarth) } \\
\quad[6,43] \\
\text { Bagging ensemble of regression trees (Treebag) [55] }\end{array}$ \\
\hline 3 & Boosting ensembles & RandomGLM $[5,65]$ \\
\hline 4 & Gaussian processes & $\begin{array}{l}\text { Gaussian processes regression with linear kernel } \\
\text { (GaussprLinear) [69] }\end{array}$ \\
\hline 5 & Generalized additive models & Generalized additive model (Gam) [70] \\
\hline 6 & $\begin{array}{l}\text { Generalized linear } \\
\text { regression }\end{array}$ & $\begin{array}{l}\text { Generalized Linear Model (Glm) [20] } \\
\text { Penalized Linear Model (Penalized) [38] }\end{array}$ \\
\hline 7 & machines & $\begin{array}{l}\text { Gradient boosting machine with linear regressors } \\
\text { (BstLm) }[9,32,50] \\
\text { Gradient boosting with regression base trees } \\
\text { (BstTree) }[9,50] \\
\text { Generalized boosting model (Gbm) }[32]\end{array}$ \\
\hline 8 & Independent Component Analysis & Independent component regression (Icr) [41] \\
\hline 9 & Least absolute shrinkage & $\begin{array}{l}\text { Least absolute shrinkage and selection operator } \\
\text { (Lasso) [73] }\end{array}$ \\
\hline 10 & Least squares & Non-negative least squares regression (NNLS) [46] \\
\hline 11 & Linear regression & Linear Model (Lm) [15] \\
\hline 12 & Neural networks & $\begin{array}{l}\text { Multi-layer perceptron (MLP) [68] } \\
\text { Averaged neural network (AvNNet) [43] } \\
\text { Generalized regression neural network (Grnn) [66] } \\
\text { Extreme learning machine (Elm) [40] } \\
\text { Deep belief neural network (Dnn) [39] } \\
\text { Elm-kernel [40] }\end{array}$ \\
\hline 13 & Other methods & $\begin{array}{l}\text { Multivariate adaptive regression (Earth) [31] } \\
\text { Projection pursuit regression (PPR) [33] }\end{array}$ \\
\hline 14 & Partial least squares & $\begin{array}{l}\text { Sparse partial least squares regression (Spls) [17] } \\
\text { Statistically Inspired Modification of PLS (Simpls) } \\
\text { [19] }\end{array}$ \\
\hline 15 & Prototype models & Cubist [56] \\
\hline 16 & Quantile regression & Rqlasso regressor (Rqlasso) [52] \\
\hline 17 & Random forests & $\begin{array}{l}\text { Random forest ensemble (Rf) [12] } \\
\text { Quantile regression forest (Qrf) [51] } \\
\text { Ensemble of extremely randomized regression trees } \\
\quad \text { (ExtraTrees) [37] }\end{array}$ \\
\hline
\end{tabular}


Table 2 continued

\begin{tabular}{lll}
\hline No. & Family & Regressors \\
\hline 18 & Regression trees & Recursive partitioning and regression tree (Rpart) \\
& & {$[13]$} \\
& & Multivariate linear tree-based model (M5) [57] \\
19 & Ridge (or Tikhonov) regression & Forward-Backward Greedy algorithm (Foba) [71] \\
20 & Support vector regression & Support vector machine for regression (Svr) [64] \\
\hline
\end{tabular}

Table 3 List of the regressors, with their tunable hyper-parameters (tried values and packages)

\begin{tabular}{|c|c|c|c|c|c|}
\hline Regressor & Hyperp. (values) & Packages & Regressor & Hyperp. (values) & Packages \\
\hline AvNNet & $\begin{array}{l}\text { size (7) } \\
\text { decay (3) }\end{array}$ & nnet & Grnn & $\operatorname{spread}(14)$ & Matlab \\
\hline Bag & - & caret & Icr & $\operatorname{ncomp}(10)$ & fastICA \\
\hline BagEarth & nprune(10) & caret & Lasso & - & elasticnet \\
\hline Bayesglm & - & arm & $\mathrm{Lm}$ & - & MASS \\
\hline BstLm & mstop (10) & bst, plyr & M5 & $\begin{array}{l}\text { pruned (2) } \\
\text { smoothed (2) } \\
\text { rules (2) }\end{array}$ & RWeka \\
\hline BstTree & mstop (4), maxdepth (5) & bst, plyr & MLP & n.hidden (20) & nnet \\
\hline Brnn & neurons (15) & brnn & NNLS & - & $\mathrm{nnls}$ \\
\hline Cubist & $\begin{array}{l}\text { committees (3) } \\
\text { neighbors (3) }\end{array}$ & Cubist & Penalized & $\lambda_{1}(5), \lambda_{2}(4)$ & penalized \\
\hline Dnn & $\begin{array}{l}\text { layer1 (10) } \\
\text { layer2 (10) } \\
\text { layer3 (10) }\end{array}$ & deepnet & PPR & nterms $(10)$ & stats \\
\hline Earth & nprune (15) & earth & Qrf & mtry (2) & quantregForest \\
\hline Elm & $\begin{array}{l}\text { nhid (20) } \\
\text { actfun (4) }\end{array}$ & elmNN & $\mathrm{Rf}$ & $\operatorname{mtry}(10)$ & randomForest \\
\hline $\begin{array}{l}\text { Elm-kernel } \\
\text { ExtraTrees }\end{array}$ & $\begin{array}{l}\sigma(25), C(25) \\
\text { mtry }(10) \\
\text { numRandomCuts }(2)\end{array}$ & $\begin{array}{l}\text { Matlab } \\
\text { extraTrees }\end{array}$ & $\begin{array}{l}\text { RandomGLM } \\
\text { Rpart }\end{array}$ & $\begin{array}{l}\text { maxInterationOrder (3) } \\
\text { complexity (10) }\end{array}$ & $\begin{array}{l}\text { randomGLM } \\
\text { rpart }\end{array}$ \\
\hline Foba & $k(2), \lambda(10)$ & foba & Rqlasso & $\lambda(10)$ & rqPen \\
\hline Gam & select (2) & gam & Simpls & ncomp (10) & pls \\
\hline GaussprLinear & $r-$ & kernlab & Spls & $K(3), \eta, \kappa(7)$ & spls \\
\hline Gbm & $\begin{array}{l}\text { n.trees (5) } \\
\text { interaction.depth (5) }\end{array}$ & gbm, plyr & Svr & $\sigma(5), C(4)$ & kernlab \\
\hline Glm & - & gbm, plyr & Treebag & - & $\begin{array}{l}\text { ipred, plyr } \\
\text { e1071 }\end{array}$ \\
\hline
\end{tabular}

the HVAC system parameters and consequently both increase user satisfaction and optimize energy consumption. We plan to address those aspects in our future work. 
Table 4 Friedman rank of the RMSE (left) and R-coefficient (right)

\begin{tabular}{|c|c|c|c|c|c|c|}
\hline \multirow[t]{2}{*}{ Order } & \multicolumn{3}{|l|}{$\underline{\text { RMSE rank }}$} & \multicolumn{3}{|c|}{$\underline{\text { R-coefficient rank }}$} \\
\hline & Regressor & Rank & MSE Avg. & Regressor & Rank & R-coefficient Avg. \\
\hline 1 & extraTrees & 1 & 0.05807 & extraTrees & 1 & 0.97052 \\
\hline 2 & $\mathrm{rf}$ & 2 & 0.06046 & $\mathrm{rf}$ & 2 & 0.96916 \\
\hline 3 & cubist & 4 & 0.06255 & cubist & 3.7 & 0.96801 \\
\hline 4 & avNNet & 4 & 0.06382 & avNNet & 4 & 0.96727 \\
\hline 5 & bstTree & 5.3 & 0.06362 & bstTree & 5.3 & 0.96738 \\
\hline 6 & elm-kernel & 5.7 & 0.06484 & elm-kernel & 5.7 & 0.96673 \\
\hline 7 & brnn & 7.7 & 0.06811 & gbm & 7.7 & 0.96595 \\
\hline 8 & $\mathrm{gbm}$ & 7.7 & 0.06635 & brnn & 8.3 & 0.96506 \\
\hline 9 & svr & 10.3 & 0.06832 & svr & 10 & 0.96505 \\
\hline 10 & qrf & 10.7 & 0,06831 & qrf & 10.7 & 0.96503 \\
\hline 11 & ppr & 11.7 & 0.07480 & ppr & 12 & 0.96154 \\
\hline 12 & bag & 13.3 & 0.07721 & bag & 13.3 & 0.96023 \\
\hline 13 & grnn & 14 & 0.07571 & grnn & 13.7 & 0.9614 \\
\hline 14 & penalized & 14.3 & 0.08885 & penalized & 14.3 & 0.95398 \\
\hline 15 & simpls & 17.7 & 0.09578 & simpls & 17.7 & 0.9503 \\
\hline 16 & mlp & 18.3 & 0.09182 & mlp & 18.3 & 0.95268 \\
\hline 17 & earth & 18.7 & 0.12496 & earth & 18.7 & 0.93754 \\
\hline 18 & rqlasso & 18.7 & 0.09719 & rqlasso & 18.7 & 0.95003 \\
\hline 19 & bagEarth & 19.3 & 0.16418 & bagEarth & 19.3 & 0.92212 \\
\hline 20 & nnls & 20.7 & 0.10783 & nnls & 20.3 & 0.94505 \\
\hline 21 & BstLm & 20.7 & 0.10757 & BstLm & 21.3 & 0.94431 \\
\hline 22 & lasso & 21.7 & 0.10847 & lasso & 21.3 & 0.94468 \\
\hline 23 & bayesglm & 25.3 & 0.12207 & bayesglm & 25 & 0.93695 \\
\hline 24 & elm & 26 & 0.11665 & $\operatorname{glm}$ & 26 & 0.93687 \\
\hline 25 & glm & 26.3 & 0.12224 & gam & 27 & 0.93687 \\
\hline 26 & spls & 27.3 & 0.22177 & spls & 27.3 & 0.89188 \\
\hline 27 & gaussprLinear & 27.3 & 0.12219 & gaussprLinear & 27.3 & 0.93688 \\
\hline 28 & gam & 27.3 & 0.12224 & elm & 27.3 & 0.93927 \\
\hline 29 & M5 & 27.7 & 0.17291 & M5 & 27.7 & 0.91652 \\
\hline 30 & $\operatorname{lm}$ & 28.3 & 0.12224 & $\operatorname{lm}$ & 28 & 0.93687 \\
\hline 31 & treebag & 29 & 0.12731 & treebag & 29 & 0.93359 \\
\hline 32 & rpart & 29.3 & 0.13357 & rpart & 29.3 & 0.92993 \\
\hline 33 & icr & 29.3 & 0.12732 & icr & 29.3 & 0.93426 \\
\hline 34 & randomGLM & 29.7 & 0.35537 & randomGLM & 29.7 & 0.84641 \\
\hline 35 & foba & 30 & 0.12355 & foba & 30 & 0.93617 \\
\hline 36 & dnn & 35.7 & 0.53150 & dnn & 35.7 & 0.70223 \\
\hline
\end{tabular}

Best algorithm amongst the whole group and has obtained the best results are shown in bold

In the performed experiments, we calculated the Friedman ranks [35] for both RMSE and R-coefficient for all regressors (see Table 4). The Friedman test is a non- 
Table 5 The best R-coefficient and RMSE are achieved by extraTrees for the forecasting horizon

\begin{tabular}{llll}
\hline & $1 \mathrm{~h}$ & $2 \mathrm{~h}$ & $3 \mathrm{~h}$ \\
\hline RMSE & 0.04041 & 0.06011 & 0.07370 \\
R-coefficient & 0.97958 & 0.96951 & 0.96245 \\
\hline
\end{tabular}

parametric statistical test. Similar to the parametric repeated measures ANOVA, it compares three or more matched or paired groups. It scores the values in each matched row in ascending order, where each row is ranked individually. It then sums the ranks in each column [34]. This test determined the actual position of each algorithm on average over all the horizons. The regressors must be sorted in a descending order based on their performance on each data set (e.g., by increasing RMSE or by decreasing Rcoefficient), and the Friedman rank of each regressor is its average position over the horizons. Figure 1 illustrates the Friedman rank for both MSE and R-coefficient in ascending order (i.e., by decreasing performance). The best results were achieved by two regressors that belong to the random forest family (ExtraTrees and RF) in both performance measurements. Generally, both figures are quite similar, with small changes in some regressor positions. Table 4 summarizes the Friedman ranks of both the MSE and the R-coefficient average for each regressor, and it clearly shows the small change in the position over all three horizons. Namely, the algorithms fall between the 24th and 28th positions and also between the Bayesian regularized neural network (Brnn) and the generalized boosting model (Gbm).

Figure 2 shows the average R-coefficient of the most reliable 20 regressors over the three prediction horizons, sorted decreasingly. The highest R-coefficients are achieved by extremely randomized regression trees (ExtraTrees) — with the accuracy R-coefficient (0.97) and the lowest RMSE average (0.058) as reported in Table 4followed by Rf, Cubist, BstTree, and AvNNet. The Figure also shows that all the algorithms that appear in the top 10 list belong to random forest family, and the accuracy obtained by Qrf is quite similar to the Bayesian model (Brnn) and Support Vector Regression (Svr). On the other hand, NNLS, Lasso, and Bstlm are at the bottom of the top 20 list, with good performance in terms of R-coefficient (around 0.94 over all horizons).

These results (ploted in Fig. 2) are quite similar to the Friedman rank of R-coefficient shown in Fig. 1. The BstTree is substituted with AvNNet, so they come in 4th and 5th position, respectively. Moreover, Bag and Grnn algorithms swap positions, becoming 12th and 13th, respectively. Regarding the last three positions, NNLS has improved its position. Unfortunately, Earth and Bagearth regressors disappeared from the top 20, while lasso and BstLm replaced them in the 19th and 20th position.

The outcomes of this comparative experiment are as follows: the extraTrees algorithm achieved the highest accuracy for the three prediction horizons in terms of Friedman rank, average values of RMSE, and R-coefficient (Table 5). ExtraTrees is less sensitive to noise and outlier values while ANN models are more sensitive, which means that extraTree is more robust. Moreover, the difference between the obtained results for three consecutive forecasting hours is quite small (around 0.01) for both R-coefficient and RMSE; this means the increase of the horizon does not rapidly 

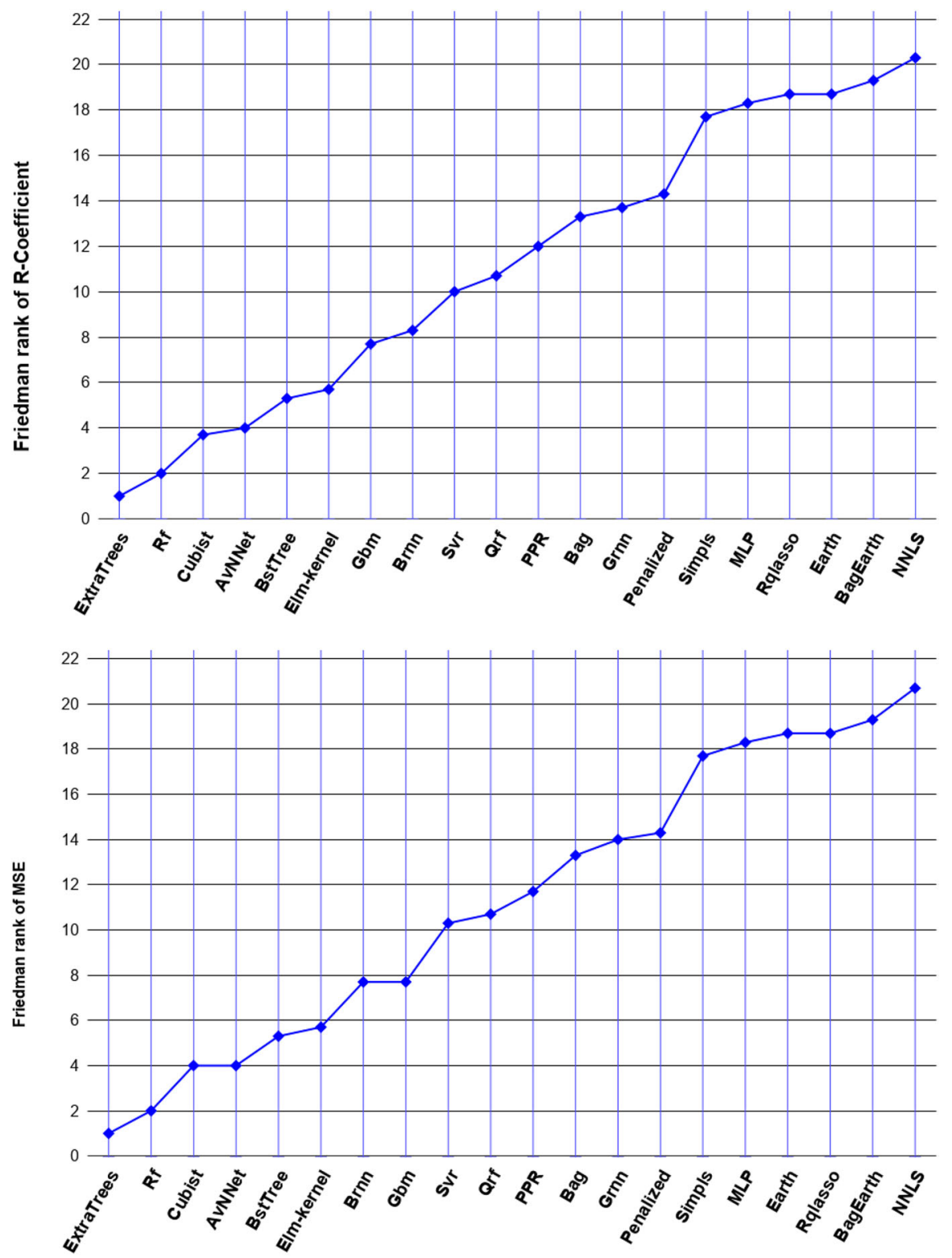

Fig. 1 Friedman rank of R-coefficient (upper panel) and RMSE (lower panel) for the 20 best regressors

affect the extraTrees accuracy. Other regressors with good performance are random forest, cubist, gradient boosting of regression trees (bstTree), average neural network committee (avNNet), and kernel ELM (elm-kernel).

There is a high agreement between average values and Friedman ranks in the results. This comparison might be useful for indoor temperature prediction for any smart build- 


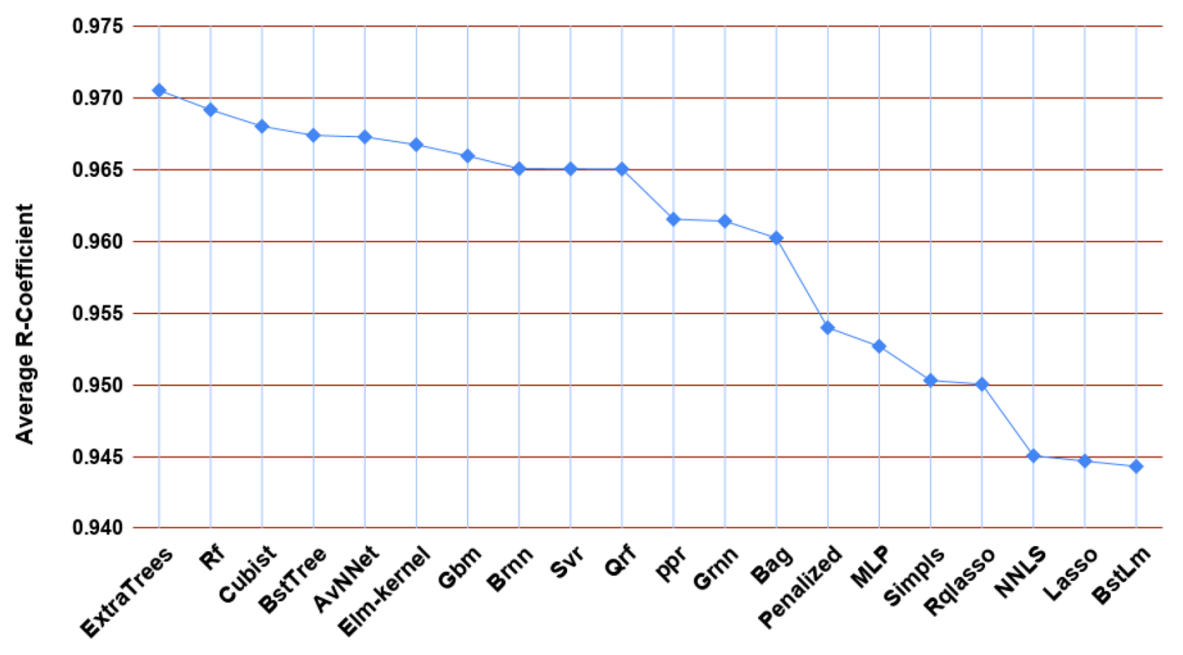

Fig. 2 Average values of R-coefficient over the data sets of the 20 best regressors to forecast three consecutive hours

ing, which facilitates building a ML forecast model to improve the energy efficiency, reduce energy consumption, and manage a building's assets.

Threats to validity A potential threat is that our results may not be valid in all HVAC systems. As we have not made any particular assumptions, and as the HVAC does not have any unique features, we believe that our results can be generalized to most other HVAC Systems. However, further research is needed to confirm this. Our study may have been internally biased from the settings of the experiments because the data was collected during winter periods in two different years with different weather conditions. Testing all algorithms using data collected during summer periods may produce different results, however, based on previous studies, the ExtraTree will obtain the best results in all scenarios [63]. Moreover, the algorithm hyper-parameters values were tuned according to the default settings shown in the Table 3 used in our study and the results are quite good. However, if we search for the optimal values of those parameters which will affect the learning process, we may get a slight improvement in the accuracy of the algorithms. The experiments were repeated 10 times to make it statistically significant, and the mean was calculated to ensure the result was correct and avoid any execution errors.

\section{Conclusions and future work}

In this paper, we compared a set of $36 \mathrm{ML}$ algorithms that belong to 20 different families to forecast the indoor temperature for three consecutive hours using real data collected from both a smart building and a weather station every 10 min. This comparison showed that the ExtraTrees algorithm performs best in terms of both the R-coefficient $(0.97 \%)$ and RMSE $(0,058 \%)$; it also ranks the highest according to the Friedman test. Other algorithms performed well are the random forest, averaged neural 
network (AvNNet), cubist, gradient boosted machines with regression trees, extreme learning machine with Gaussian kernels, and support vector machine for regression. The outcomes of this study show that the extraTrees is more robust to outliers and data noise, while most of the algorithms such as ANN are highly sensitive to data noise. Furthermore, increasing the forecasting time does not decrease the accuracy of the best model. We found that the difference between the obtained results for three consecutive forecasting hours is insignificant (around 0.01) for both R-coefficient and RMSE; this means that the increase of the horizon does not rapidly affect the accuracy of extraTrees. Finally, it is possible to use a standard ML algorithm to forecast the indoor temperature with reasonable accuracy based on weather and sensors data linked to the smart building.

However, more research efforts should be made in the future to optimize the HVAC parameters based on the prediction of the indoor temperature. Researchers need to consider the following: integrating an incremental training and online learning approach to improve the accuracy and the robustness of the identified model. Real time user feedback during the deployment phase (Interactive learning) for new data behavior that will help in improving model efficiency. Raising the forecast horizon for longer time periods (days ahead), considering user satisfaction (comfort level), and energy consumption. Integrating the winner model (ExtraTree) with building management systems and predicting in real-time. Validating the results in other buildings using other sensor data. Finally, addressing possible noise or missing data linked to sensor failure scenarios during the run time.

Acknowledgements Open access funding provided by Malmö University.

Open Access This article is licensed under a Creative Commons Attribution 4.0 International License, which permits use, sharing, adaptation, distribution and reproduction in any medium or format, as long as you give appropriate credit to the original author(s) and the source, provide a link to the Creative Commons licence, and indicate if changes were made. The images or other third party material in this article are included in the article's Creative Commons licence, unless indicated otherwise in a credit line to the material. If material is not included in the article's Creative Commons licence and your intended use is not permitted by statutory regulation or exceeds the permitted use, you will need to obtain permission directly from the copyright holder. To view a copy of this licence, visit http://creativecommons.org/licenses/by/4.0/.

\section{References}

1. Alawadi, S., Delgado, M.F., Pérez, D.M.: Machine learning algorithms for pattern visualization in classification tasks and for automatic indoor temperature prediction. Ph.D. thesis, Universidade de Santiago de Compostela (2018)

2. Alawadi, S., Mera, D., Fernández-Delgado, M., Taboada, J.A.: Comparative study of artificial neural network models for forecasting the indoor temperature in smart buildings. In: 2017 2nd International Conference on Smart Cities, pp. 29-38. Springer (2017)

3. Aliberti, A., Bottaccioli, L., Macii, E., Di Cataldo, S., Acquaviva, A., Patti, E.: A non-linear autoregressive model for indoor air-temperature predictions in smart buildings. Electronics 8(9), 979 (2019)

4. Alzubi, J., Nayyar, A., Kumar, A.: Machine learning from theory to algorithms: an overview. In: 2018 2nd National Conference on Computational Intelligence (NCCI): Journal of Physics, vol. 1142, p. 012012. IOP Publishing (2018)

5. Alzubi, J.A.: Diversity based improved bagging algorithm. In: 2015 1st Proceedings of The International Conference on Engineering \& MIS (ICEMIS) 2015, pp. 35-40. ACM (2015)

6. Alzubi, J.A.: Diversity-based boosting algorithm. Int. J. Adv. Comput. Sci. Appl.7(5), 524-529 (2016) 
7. Alzubi, J.A.: Research article optimal classifier ensemble design based on cooperative game theory. Res. J. Appl. Sci. Eng. Technol. 11(12), 1336-1343 (2015)

8. Attoue, N., Shahrour, I., Younes, R.: Smart building: use of the artificial neural network approach for indoor temperature forecasting. Energies 11(2), 395 (2018)

9. ATLAS Collaboration et al.: The evolution of boosting algorithms-from machine learning to statistical modelling. Methods Inf. Med. 53(6), 419-427 (2014)

10. Ben-Nakhi, A.E., Mahmoud, M.A.: Cooling load prediction for buildings using general regression neural networks. Energy Convers. Manag. 45(13), 2127-2141 (2004)

11. Breiman, L.: Bagging predictors. Mach. Learn. 24, 123-140 (1996)

12. Breiman, L.: Random forests. Mach. Learn. 45, 5-32 (2001)

13. Breiman, L., Friedman, J., Olshen, R.A., Stone, C.J.: Classification and Regression Trees. Wadsworth and Brooks, Pacific Grove (1984)

14. Catalina, T., Virgone, J., Blanco, E.: Development and validation of regression models to predict monthly heating demand for residential buildings. Energy Build. 40(10), 1825-1832 (2008)

15. Chambers, J.M.: Linear models, chapter 4. In: Chambers, J.M., Hastie, T.J. (eds.) Wadsworth \& Brooks/Cole (1992)

16. Chen, M., Hao, Y., Hwang, K., Wang, L., Wang, L.: Disease prediction by machine learning over big data from healthcare communities. IEEE Access 5, 8869-8879 (2017)

17. Chun, H., Keles, S.: Sparse partial least squares for simultaneous dimension reduction and variable selection. J. R. Stat. Soc. 72, 3-25 (2010)

18. Chengliang, X., Chen, H., Wang, J., Guo, Y., Yuan, Y.: Improving prediction performance for indoor temperature in public buildings based on a novel deep learning method. Build. Environ. 148, 128-135 (2019)

19. De Jong, S.: SIMPLS: an alternative approach to partial least squares regression. Chemom. Intell. Lab. Syst. 18, 251-263 (1993)

20. Dobson, A.J.: An Introduction to Generalized Linear Models. Chapman and Hall, London (1990)

21. Dong, B., Cao, C., Lee, S.E.: Applying support vector machines to predict building energy consumption in tropical region. Energy Build. 37(5), 545-553 (2005)

22. Doukas, H., Patlitzianas, K.D., Iatropoulos, K., John, P.: Intelligent building energy management system using rule sets. Build. Environ. 42(10), 3562-3569 (2007)

23. Dounis, A.I., Caraiscos, C.: Advanced control systems engineering for energy and comfort management in a building environment a review. Renew. Sustain. Energy Rev. 13(6), 1246-1261 (2009)

24. Ekici, B.B., Aksoy, U.T.: Prediction of building energy consumption by using artificial neural networks. Adv. Eng. Softw. 40(5), 356-362 (2009)

25. Erickson, V.L., Carreira-Perpiñán, M.Á., Cerpa, A.E.: OBSERVE: Occupancy-based system for efficient reduction of HVAC energy. In: Proceedings of the 2011 10th ACM/IEEE International Conference on Information Processing in Sensor Networks, pp. 258-269. IEEE (2011)

26. EU. Life-OPERE. http://www.life-opere.org/en. Accessed June 2019

27. Fernandez-Delgado, M., Sirsat, M.S., Cernadas, E., Alawadi, S., Barro, S., Febrero-Bande, M.: An extensive experimental survey of regression methods. Neural Netw. 111, 11-34 (2018)

28. Fernández-Delgado, M., Cernadas, E., Barro, S., Amorim, D.: Do we need hundreds of classifiers to solve real world classification problems? J. Mach. Learn. Res. 15(1), 3133-3181 (2014)

29. Fong, K.F., Hanby, V.I., Chow, T.-T.: Hvac system optimization for energy management by evolutionary programming. Energy Build. 38(3), 220-231 (2006)

30. Foresee, F.D., Hagan, M.T.: Gauss-Newton approximation to Bayesian regularization. In: International Joint Conference on Neural Networks, pp. 1930-1935 (1997)

31. Friedman, J.H.: Multivariate adaptive regression splines. Ann. Stat. 19(1), 1-141 (1991)

32. Friedman, J.H.: Greedy function approximation: a gradient boosting machine. Ann. Stat. 29, 1189$1232(2001)$

33. Friedman, J.H., Stuetzle, W.: Projection pursuit regression. J. Am. Stat. Assoc. 76(376), 817-823 (1981)

34. Friedman, M.: The use of ranks to avoid the assumption of normality implicit in the analysis of variance. J. Am. Stat. Assoc. 32(200), 675-701 (1937)

35. García, S., Fernández, A., Benítez, A.D., Herrera, F.: Statistical comparisons by means of nonparametric tests: a case study on genetic based machine learning. In: Proceedings of the II Congreso Español de Informática (CEDI 2007). V Taller Nacional de Minería de Datos y Aprendizaje (TAMIDA), pp. 95-104 (2007) 
36. Gelman, A., Jakulin, A., Pittau, M.G., Su, Y.S.: A weakly informative default prior distribution for logistic and other regression models. Ann. Appl. Stat. 2(4), 1360-1383 (2009)

37. Geurts, P., Ernst, D., Wehenkel, L.: Extremely randomized trees. Mach. Learn. 63(1), 3-42 (2006)

38. Goeman, J.J.: L-1 penalized estimation in the cox proportional hazards model. Biom. J. 52, 70-84 (2010)

39. Hinton, G.E., Osindero, S., Teh, Y.W.: A fast learning algorithm for deep belief nets. Neural Comput. 18(7), 1527-1554 (2006)

40. Huang, G.-B., Zhou, H., Ding, X., Zhang, R.: Extreme learning machine for regression and multiclass classification. IEEE Trans. Syst. Man Cybern. Part B Cybern. 42(2), 513-529 (2012)

41. Hyvarinen, A., Oja, E.: Independent component analysis: algorithms and applications. Neural Netw. 13, 411-430 (2000)

42. Jin, J., Shu, S., Lin, F.: Prediction of indoor air temperature based on deep learning. Sens. Mater. 31(6), 2029-2042 (2019)

43. Kuhn, M., et al.: Building predictive models in $r$ using the caret package. J. Stat. Softw. 28(5), 1-26 (2008)

44. Kwok, S.S.K., Yuen, R.K.K., Lee, E.W.M.: An intelligent approach to assessing the effect of building occupancy on building cooling load prediction. Buil. Environ. 46(8), 1681-1690 (2011)

45. Langley, P., Simon, H.A.: Applications of machine learning and rule induction. Commun. ACM 38(11), 54-64 (1995)

46. Lawson, C.L., Hanson, R.J.: Solving least squares problems, vol. 15 of Classics in Applied Mathematics. Society for Industrial and Applied Mathematics (SIAM) (1995)

47. Li, K., Hongye, S., Chu, J.: Forecasting building energy consumption using neural networks and hybrid neuro-fuzzy system: a comparative study. Energy Build. 43(10), 2893-2899 (2011)

48. Li, Q., Meng, Q., Cai, J., Yoshino, H., Mochida, A.: Applying support vector machine to predict hourly cooling load in the building. Appl. Energy 86(10), 2249-2256 (2009)

49. MacKay, D.J.C.: Bayesian interpolation. Neural Comput. 4, 415-447 (1992)

50. Maloney, K.O., Schmid, M., Weller, D.E.: Applying additive modelling and gradient boosting to assess the effects of watershed and reach characteristics on riverine assemblages. Methods Ecol. Evol. 3(1), 116-128 (2012)

51. Meinshausen, N.: Quantregforest: quantile regression forests. R package version 0.2-2 (2007)

52. Mizera, I., Koenker, R.: Convex optimization in r. J. Stat. Softw. 60(5), 1-23 (2014)

53. Moroşan, P.-D., Bourdais, R., Dumur, D., Buisson, J.: Building temperature regulation using a distributed model predictive control. Energy Build. 42(9), 1445-1452 (2010)

54. Nguyen, T.A., Aiello, M.: Energy intelligent buildings based on user activity: a survey. Energy Build. 56, 244-257 (2013)

55. Peters, A., Hothorn, T., Lausen, B.: ipred: Improved predictors. R News 2(2), 33-36 (2002)

56. Quinlan, R.: Combining instance-based and model-based learning. In Proc. Intl. Conf. on Machine Learning, pp. 236-243 (1993)

57. Quinlan, J.R. et al.: Learning with continuous classes. In: 1992 5th Australian Joint conference on Artificial Intelligence, vol. 92, pp. 343-348. World Scientific (1992)

58. Ríos-Moreno, G.J., Trejo-Perea, M., Castaneda-Miranda, R., Hernández-Guzmán, V.M., Herrera-Ruiz, G.: Modelling temperature in intelligent buildings by means of autoregressive models. Autom. Constr. 16(5), 713-722 (2007)

59. Rodriguez-Mier, P., Fresquet, M., Mucientes, M., Bugarın, A.: Prediction of indoor temperatures for energy optimization in buildings. In: Conference of the Spanish Association for Artificial Intelligence, pp. 675-684 (2016)

60. Rosenzweig, C., Solecki, W., Hammer, S.A., Mehrotra, S.: Cities lead the way in climate-change action. Nature 467(7318), 909 (2010)

61. Santin, O.G., Itard, L., Visscher, H.: The effect of occupancy and building characteristics on energy use for space and water heating in dutch residential stock. Energy Build. 41(11), 1223-1232 (2009)

62. Shanthamallu, U.S., Spanias, A., Tepedelenlioglu, C., Stanley, M.: A brief survey of machine learning methods and their sensor and iot applications. In: 2017 8th International Conference on Information, Intelligence, Systems and Applications (IISA), pp. 1-8. IEEE (2017)

63. Sirsat, M.S., García, E.C., Delgado, M.F.: Application of machine learning to agricultural soil data. Ph.D. thesis, Universidade de Santiago de Compostela (2017)

64. Smola, A.J., Schölkopf, B.B.: A tutorial on support vector regression. Stat. Comput. 14(3), 199-222 (2004) 
65. Song, L., Langfelder, P., Horvath, S.: Random generalized linear model: a highly accurate and interpretable ensemble predictor. BMC Bioinform. 14(1), 1-22 (2013)

66. Specht, D.F.: A general regression neural network. IEEE Trans. Neural Netw. 2, 568-576 (1991)

67. Sülo, Idil, K., Seref, R., Dogan, G., Brown, T.: Energy efficient smart buildings: LSTM neural networks for time series prediction. In: 2019 International Conference on Deep Learning and Machine Learning in Emerging Applications (Deep-ML), pp. 18-22. IEEE (2019)

68. Tang, J., Deng, C., Huang, G.-B.: Extreme learning machine for multilayer perceptron. IEEE Trans. Neural Netw. Learn. Syst. 27(4), 809-821 (2015)

69. Williams, C.K.I., Barber, D.: Bayesian classification with Gaussian processes. IEEE Trans. Pattern Anal. Mach. Intell. 20(12), 1342-1351 (1998)

70. Wood, S.N.: Fast stable restricted maximum likelihood and marginal likelihood estimation of semiparametric generalized linear models. J. R. Stat. Soc. 1(73), 3-36 (2011)

71. Zhang, T.: Adaptive forward-backward greedy algorithm for learning sparse representations. IEEE Trans. Inf. Theor. 57(7), 4689-4708 (2011)

72. Zhao, H., Magoulès, F.: A review on the prediction of building energy consumption. Renew. Sustain. Energy Rev. 16(6), 3586-3592 (2012)

73. Zou, H., Hastie, T.: Regularization and variable selection via the elastic net. J. R. Stat. Soc. Ser B (Stat. Methodol.) 67(2), 301-320 (2005)

Publisher's Note Springer Nature remains neutral with regard to jurisdictional claims in published maps and institutional affiliations.

\section{Affiliations}

\section{Sadi Alawadi ${ }^{1}$ - David Mera ${ }^{2}$ - Manuel Fernández-Delgado ${ }^{2}$. Fahed Alkhabbas ${ }^{1} \cdot$ Carl Magnus Olsson ${ }^{1} \cdot$ Paul Davidsson $^{1}$}

David Mera

david.mera@usc.es

Manuel Fernández-Delgado

manuel.fernandez.delgado@usc.es

Fahed Alkhabbas

fahed.alkhabbas@mau.se

Carl Magnus Olsson

carl.magnus.olsson@mau.se

Paul Davidsson

paul.davidsson@mau.se

1 Internet of Things and People Research Center Department of Computer Science and Media Technology, Malmö University, 20506 Malmö, Sweden

2 Centro Singular de Investigación en Tecnoloxías da Información (CiTIUS), Universidade de Santiago de Compostela, Santiago de Compostela, Spain 